\title{
Membrana amniótica no tratamento dos afinamentos corneais e esclerais
}

\author{
Amniotic membrane in the treatment of corneal and scleral thinning
}

\author{
Daniella Fairbanks ${ }^{1}$ \\ Luiz Antônio Vieira² \\ Walner Daros dos Santos ${ }^{3}$ \\ Greicie Cristina Guerra Attie $^{3}$ \\ José Álvaro Pereira Gomes ${ }^{4}$ \\ Denise de Freitas ${ }^{5}$
}

\begin{tabular}{|l|}
\hline RESUMO \\
\hline Objetivo: Investigar a utilização da membrana amniótica como adjuvante \\
no tratamento e restabelecimento de espessura dos afinamentos córneo- \\
esclerais e epitelização corneal. Métodos: A membrana amniótica foi \\
captada a partir de parto cesáreo e conservada em meio de preservação de \\
córnea e glicerol 1:1 e conservada a - $80^{\circ}$ C. Sete olhos de 7 pacientes, sendo \\
4 portadores de afinamento corneal por afecções neurotróficas (Grupo 1: \\
2 herpes simples vírus; 1 após transplante de córnea; 1 por radioterapia) \\
e 3 portadores de afinamento escleral após exérese de pterígio (Grupo $2:$ \\
2 com beta-terapia e 1 sem beta-terapia) foram submetidos à cirurgia para \\
restabelecimento da superfície ocular e espessura córneo-escleral empre- \\
gando membrana amniótica. Resultados: O tempo médio de seguimento \\
foi de 12 meses (variação entre 11 meses e 15 meses). Um caso de úlcera \\
neurotrófica secundária a radioterapia apresentou insucesso. Obtivemos \\
sucesso do restabelecimento da superfície ocular e da espessura nos \\
outros 6 casos. Em relação à acuidade visual, 1 caso obteve melhora e os \\
outros 6 permaneceram inalterados. A média de tempo de epitelização foi \\
de $26,6 \pm 5,8$ dias para o grupo 1 e $10,6 \pm 4,0$ dias para o grupo 2. Conclusões: \\
O uso de membrana amniótica constitui opção alternativa de grande \\
utilidade na reconstrução da superfície ocular dos casos de afinamento \\
córneo-escleral. Estudos com maior casuística e tempo de seguimento são \\
necessários para melhor avaliar esse procedimento. \\
\hline
\end{tabular}

Descritores: Âmnio/transplante; Transplante de tecido fetal; Preservação de tecido; Transplante homólogo; Córnea/cirurgia; Córnea/patologia; Conjuntiva/transplante; Esclera/ cirurgia, Epitélio da córnea; Seguimentos

Palo - UNIFESP e da Faculdade de Medicina do $\mathrm{ABC}$ - FUABC.

Coutor e Colaborador do Setor de Doenças Externas Córnea do Depto. de Oftalmologia da Universidade Federal de São Paulo - UNIFESP.

${ }^{3}$ Colaborador(a) do Setor de Doenças Externas e Córnea do Depto. de Oftalmologia da Universidade Federal de São Paulo - UNIFESP.

${ }^{4}$ Doutor em Oftalmologia e Colaborados do Setor de Doenças Externas e Córnea do Depto. de Oftalmologia da Universidade Federal de São Paulo - UNIFESP.

${ }^{5}$ Livre Docente em Oftalmologia e Colaboradora do Setor de Doenças Externas e Córnea do Depto. de Oftalmologia da Universidade Federal de São Paulo - UNIFESP.

Endereço para correspondência: Rua Domingos Fernandes, 144 - São Paulo (SP) CEP 04509-010.

E-mail: dfairbanks@ig.com.br

danifairbanks@hotmail.com

Recebido para publicação em 27.04.2001 Aceito para publicação em 12.06.2002

Nota Editorial: Pela análise deste trabalho e por sua anuência sobre a divulgação desta nota, agradecemos ao Dr. Sérgio Kwitko.

\section{INTRODUÇÃO}

\begin{tabular}{l} 
INTRODUÇÃo \\
\hline A membrana amniótica é composta por uma membrana basal espessa, \\
formada basicamente de colágeno tipo IV e laminina, e uma matriz estromal \\
avascular ${ }^{(1-3)}$. Na área cirúrgica, tem sido utilizada como enxerto nas queima- \\
duras de pele, na reparação de onfalocele e na prevenção de adesão tecidual \\
em cirurgias da cabeça, abdômen, pélvis, vagina e de laringe ${ }^{(1-3)}$. \\
Sua utilização baseia-se na capacidade de beneficiar o processo de \\
epitelização por facilitar a adesão e migração das células epiteliais basais, \\
prevenir a apoptose e restaurar o fenótipo epitelial ${ }^{(3)}$. Além disso, reduz os \\
processos inflamatórios, angiogênico e cicatricial e possui documentada \\
ação antimicrobiana ${ }^{(1-5)}$. Por não expressar os antígenos de histocompatibi- \\
lidade HLA-A, B ou DR, a membrana amniótica preservada é considerada \\
imunologicamente inerte, o que a torna uma excelente opção de enxerto ${ }^{(1,4,6)}$. \\
Mais recentemente, o transplante da membrana amniótica foi reintrodu- \\
zido na reconstrução da superfície ocular nos casos de doenças cicatriciais
\end{tabular}


da córnea e conjuntiva, tais como queimadura, penfigóide cicatricial, eritema multiforme ${ }^{(4,7-10)}$; na promoção da cicatrização nos defeitos epiteliais persistentes ${ }^{(11)}$; como enxerto após a remoção cirúrgica de pterígio e tumores ${ }^{(12-13)}$; no tratamento de bolhas filtrantes com vazamento ${ }^{(14)}$; redução da opacidade corneal pós ceratectomia fototerapêutica ${ }^{(15)}$; como procedimento inicial antes de transplante de limbo nos casos de deficiência de células germinativas limbares ${ }^{(7)}$; e nas reconstruções palpebrais ${ }^{(13)}$.

Devido à fácil obtenção e documentada utilidade em oftalmologia, a membrana amniótica representa uma opção alternativa a ser mais bem estudada para o tratamento de afecções da superfície ocular. O objetivo deste trabalho é avaliar o uso de membrana amniótica para o tratamento dos afinamentos de córnea e esclera e também sua influência na epitelização da córnea.

\section{MÉTODOS}

\section{Obtenção e preparação da membrana amniótica}

A obtenção, preparação e preservação da membrana amniótica foram realizadas de acordo com as normas do protocolo aprovado pela Comissão de Ética da UNIFESP. Essas normas foram baseadas no protocolo proposto por Tseng et al. ${ }^{(3,7)}$, seguindo as premissas impostas pela "United States Food and Drug Administration (FDA)" $\mathrm{e}$ "The American Association of Tissue Banking (AATB)"(16-18).

A membrana amniótica foi obtida a partir de placentas provenientes de cesáreas eletivas de pacientes do Hospital e Maternidade São Luiz, após consentimento pelas gestantes e pelos obstetras. Todas essas pacientes tinham exames sorológicos negativos para HIV-1, Hepatite B (HBsAg) e sífilis (VDRL), que foram re-confirmados pela realização de sorologia do sangue do cordão umbilical após o parto.

Após a obtenção da placenta no centro cirúrgico obstétrico, procedeu-se à sua lavagem com soro fisiológico $0,9 \% \mathrm{em}$ ambiente estéril. Manualmente, separou-se o âmnio do córion. O âmnio foi lavado com solução tampão fosfato até que se retirasse todos os coágulos aderidos e depois também lavados com solução antibiótica contendo 1000U/ml de penicilina, $20 \mathrm{mcg} / \mathrm{ml}$ de estreptomicina e $2,5 \mathrm{mcg} / \mathrm{ml}$ de anfotericina B sendo deixada por 5 minutos por 3 vezes em imersão. Após essa lavagem, o âmnion foi estendido por sobre um filtro de nitrocelulose estéril (Millipore, Bedfort, MA, EUA) com a face epitelial para cima. A membrana aderida ao filtro foi cortada em fragmentos que variavam de $2 \times 2 \mathrm{~cm}$ a $4 \times 4 \mathrm{~cm}$, colocados em um recipiente estéril contendo glicerol (Baxter Healthcare Corporation, Stone Mountain, GA, EUA) e meio de preservação de córnea TC-199 modificado (Ophthalmos, São Paulo/SP, Brasil) na proporção de $1: 1$ e congelados a $-80^{\circ} \mathrm{C}$. Amostras de cada membrana obtida foram enviadas para estudo anatomopatológico e microbiológico (bacterioscopia e cultura) para controle de qualidade. O tempo máximo para utilização das membranas foi de 4 meses após a congelação.

\section{Pacientes}

O grupo de estudo incluiu 7 olhos de 7 pacientes consecutivos, sendo 4 portadores de úlcera neurotrófica (Grupo 1: 2 por Herpes Simples Vírus (HSV), 1 pós-radioterapia por linfoma não Hodgkin, 1 pós-transplante de córnea (TX) e 3 pacientes com afinamento escleral após cirurgia de pterígio sendo 2 com betaterapia (grupo 2). Todos foram submetidos à cirurgia reconstrutiva da superfície ocular empregando membrana amniótica. Seis pacientes $(85,71 \%)$ eram do sexo feminino e 1 do sexo masculino (14,29\%). A idade média era 64,28 anos (variação entre 53 e 84 anos).Os detalhes das características dos pacientes encontram-se especificados na tabela 1.

Todos os pacientes foram devidamente instruídos e assinaram um detalhado termo de consentimento sobre o tratamento a que seriam submetidos.

\section{Pré-operatório}

Os pacientes foram submetidos a exame oftalmológico completo, composto de avaliação da melhor acuidade visual corrigida, biomicroscopia, tonometria (aplanação ou Tonopen), fundo de olho (quando possível) e avaliação de sensibilidade de córnea com filamento de algodão de cotonete. A avaliação da superfície ocular obedeceu a critérios de exame biomicroscópico detalhado, com e sem coloração por fluoresceína sódica $1 \%$. Avaliou-se o padrão da superfície ocular (transparência, regularidade, presença de queratinização e porcentagem de afinamento), presença de neovascularização e o tipo de coloração por fluoresceína. Para melhor documentar o exame préoperatório, foram realizadas fotografias na lâmpada de fenda.

Grupo 1: Os pacientes com úlcera neurotrófica por herpes (caso 1 e 2) foram tratados com Valaciclovir (Glaxo Smithkline, Brasil) $500 \mathrm{mg}$ 12/12h administrado oralmente e instilação de metilcelulose $0,5 \%$ tópico sem preservativo $3 / 3 \mathrm{~h}$ (Ophthalmos, Brasil) por mais de 8 semanas, sem melhora do quadro clínico. O caso 3 foi submetido ao uso de lente de contato terapêutica com cobertura antibiótica tópica com ofloxacina $0,3 \%$ (Allergan, Brasil) na tentativa de melhorar o defeito epitelial por 15 dias. O caso 4 foi submetido ao uso de adesivo tecidual, que permaneceu por um período de 3 meses. Em relação aos portadores de afinamento escleral (Grupo 2), incluímos apenas casos relacionados com cirurgia de pterígio submetidos ou não à betaterapia. Só foram submetidos à cirurgia de membrana no Grupo 2 os casos que apresentavam risco de perfuração ocular (instituiu-se indicação de cirurgia quando o afinamento escleral apresentava espessura igual ou menor que $10 \%$ da espessura escleral normal de área vizinha à lesão, medido à lâmpada de fenda).

\section{Técnica cirúrgica e pós-operatório}

A técnica cirúrgica utilizada no grupo 1 foi semelhante à descrita por Kruse et al. ${ }^{(19)}$. A técnica utilizada nos pacientes do Grupo 2 baseou-se numa adaptação da técnica acima realizada sobre a episclera. Todas cirurgias foram realizadas sob anestesia local, tópica nos grupos 1 e 2 (cloridrato de proximetacaína $5 \mathrm{mg}$, Allergan) e subconjuntival no grupo 2 (xilocaína com 
epinefrina 1:200.000, AstraZeneca). Cada grupo descrito foi operado por um cirurgião (L.A.V. grupo 1 e D.V.B.F. grupo 2). No grupo 1 foi realizada cuidadosa remoção de debris celulares na base e borda da úlcera com cotonetes. O epitélio na área de 1 a $2 \mathrm{~mm}$ ao redor da úlcera foi retirado com lâmina 15 (Becton Dickinson, Brasil). Essa área serviu como base para melhor coaptação da membrana, permitindo a migração do epitélio sobre ela. No grupo 2, foi realizada liberação da conjuntiva ao redor da área afinada, seguida de debridamento do epitélio sobrejacente, quando presente. Foi também realizada delaminação da episclera, na borda do afinamento, com lâmina 15, formando um sulco para a fixação das membranas para preencher o afinamento.

Após ser descongelada, a membrana amniótica foi lavada com solução salina balanceada (BSS) por 5 minutos e colocada sobre o olho, mantendo a orientação da face da membrana basal para cima. Em seguida, confeccionou-se fragmentos de membrana do tamanho da lesão, que foram colocados na base da área afinada.

Nos dois grupos foram colocadas 2 ou 3 camadas de tecido na área afinada, de acordo com o grau de afinamento. No grupo 1 , as primeiras camadas de membrana amniótica não foram suturadas na córnea. No grupo 2, realizou-se sutura com Vicryl 8.0. Finalmente, um fragmento de membrana de tamanho ligeiramente maior que a lesão $( \pm 2 \mathrm{~mm})$ foi colocado sobre toda a área desepielizada.O tecido foi fixado tanto na córnea (grupo 1) como na episclera (grupo 2) com pontos separados de nylon 10.0, tendo sido sepultados quando possível. No caso 3, foi realizada tarsorrafia temporal. Avaliação oftalmológica completa foi repetida semanalmente no grupo 1 , nos primeiros 2 meses, e depois quinzenalmente até o $4^{\circ}$ mês de pós-operatório. No grupo 2, essas avaliações foram realizadas no $1^{\circ}$ dia de pós-operatório, $1^{\underline{a}}$ semana e depois mensalmente até essa data.

Ofloxacina 0,3\% 6/6 horas e Dexametasona $0,005 \%$ de 2 a 4 vezes ao dia foram instiladas topicamente conforme o caso e mantidos por 30 dias no grupo 1 . Nesses casos, fez-se uso de lente de contato terapêutica (LCT). No grupo 2, foi indicado associação antibiótica tópica de Tobramicina $3 \mathrm{mg} / \mathrm{ml}$ e Dexametasona $1 \mathrm{mg} / \mathrm{ml}$ a cada 4 horas, que foi descontinuada progressivamente conforme a inflamação observada. Foram utilizadas medicações diferentes nos 2 grupos pois iniciamos o trabalho com o grupo 2 introduzindo aminoglicosídio e posteriormente quando criamos o grupo 1 e resolvemos avaliar a epitelização, trocamos de antibiótico devido a sua toxicidade epitelial.

$\mathrm{O}$ caso 5 era portador de glaucoma e fazia uso de Maleato de Timolol 0,5\% (Merck, Sharp \& Dohme, Brasil) de 12/12horas e Pilocarpina 2\% (Allergan, Brasil) 4 vezes ao dia, que não foi suspensa durante o trabalho.

\section{Critérios de avaliação dos resultados}

Para fins didáticos, classificou-se o resultado em 3 categorias: 1) Sucesso (S): reconstrução da superfície ocular bemsucedida, com cicatrização do epitélio e restabelecimento de espessura corneal ou escleral; 2) Sucesso Parcial (SP): reconstituição da superfície ocular, com cicatrização de defeito epitelial corneal ou escleral restabelecendo parte da espessura; 3) Insucesso (I): reconstrução da superfície ocular malsucedida, com recorrência de defeito epitelial. Esses pacientes necessitaram de outro procedimento cirúrgico para conter processo inflamatório. A avaliação da espessura corneal e escleral foi realizada com lâmpada de fenda.

\section{RESULTADOS}

O tempo médio de seguimento dos pacientes foi de 12 meses (variação entre 11 e 15 meses).

Três casos do grupo 1 (75\%) e dois do grupo $2(66,7 \%)$ apresentaram sucesso total com restabelecimento da espessura tanto da córnea quanto da esclera, além de completa epitelização (Figuras 1 e 2, Tabela 1).

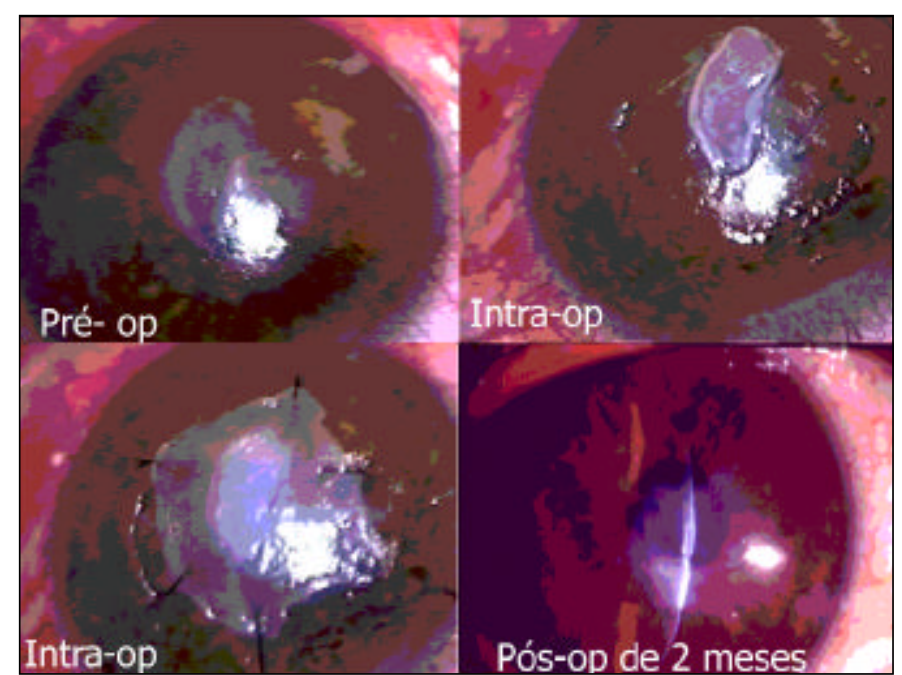

Figura 1 - (Caso 2): Pré,intra e pós-operatório de 2 meses de úlcera neurotrófica por Herpes simples vírus

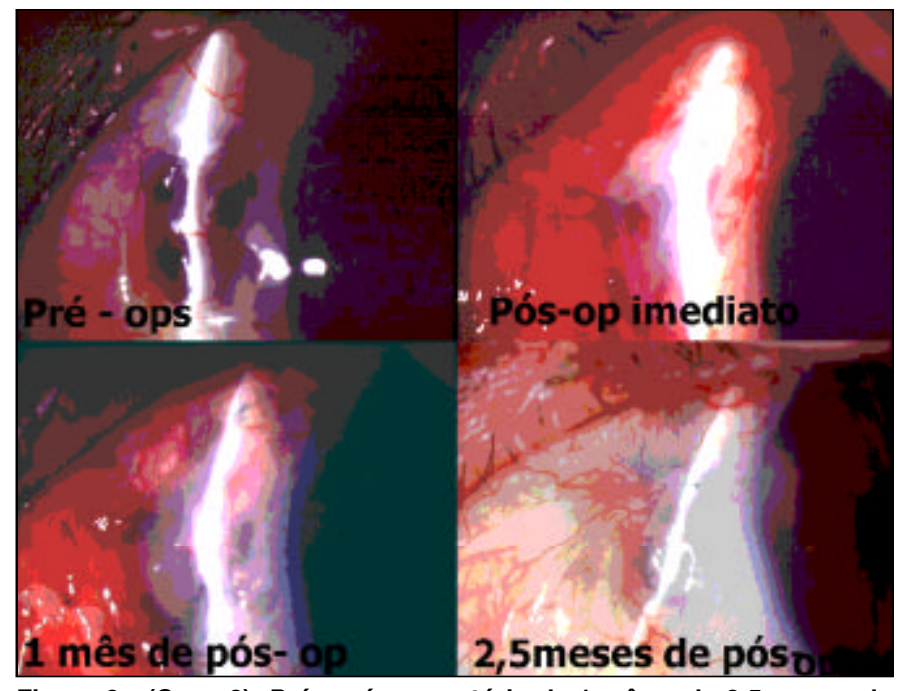

Figura 2 - (Caso 6): Pré e pós-operatório de 1 mês e de 2,5 meses de afinamento escleral pós cirurgia de pterígio e beta-terapia 
Um caso (33,3\%) no grupo 2 apresentou sucesso parcial, com restabelecimento de espessura em apenas parte da lesão. Um caso (25\%) do grupo 1 (caso 3 - úlcera trófica pós-radioterapia) apresentou insucesso, tendo sido submetido a várias cirurgias sem restabelecimento da espessura nem da epitelização.

Em relação à acuidade visual, obsservamos que seis casos $(85,7 \%)$ permaneceram inalterados e 1 caso de afinamento corneal central $(14,3 \%)$ apresentou melhora, provavelmente pela regularização da superfície após a cirurgia.

A média da espessura corneal do Grupo 1 foi de 27,5 \pm $12,6 \%$ no pré-operatório e de $62,5 \pm 35,9 \%$ no pós-operatório. No Grupo 2, a média da espessura escleral pré-operatório foi $10 \pm 0 \%$ e pós-operatório $78,3 \pm 18,9 \%$ (Tabela1).

A média de tempo de epitelização foi de $26,6 \pm 5,8$ dias para o grupo 1 e 10,6 $\pm 4,0$ dias para o grupo 2. Foi excluído dessa média o caso 3 , que não apresentou epitelização.

\section{DISCUSSÃO}

Em 1995, Kim e Tseng reintroduziram o uso de membrana amniótica em cirurgia oftalmológica num modelo experimental de queimadura, com bons resultados na recuperação da superfície ocular ${ }^{(3)}$. Esses resultados podem ser explicados pelas características inatas da membrana amniótica, que possui uma membrana basal espessa e propriedades antiinflamatórias, antiapoptóticas e inibitórias da cicatrização, provavelmente relacionadas à presença e/ou inibição de fatores de crescimento, tais como TGF-beta 1 e 2, bFGF e $\mathrm{HGF}^{(3-4,7-8,13,16)}$. Estudos recentes demonstraram que o uso da membrana amniótica possibilita a restauração de um meio-ambiente estromal perilimbar sem inflamação ${ }^{(7,18)}$.

Os primeiros resultados cirúrgicos em humanos foram publicados logo em seguida. Shimazaki et al. utilizaram transplante de membrana amniótica e de limbo autólogo ou alógeno na reconstrução da superfície ocular de 7 olhos com queimaduras químicas e térmicas, com resultados animadores ${ }^{(8)}$. Tseng et al. relataram bons resultados com o uso de membrana amniótica na reconstrução da superfície conjuntival após remoção de lesões conjuntivais e simbléfaro $^{(13)}$. Gomes et al. apresentaram bons resultados na reconstrução da superfície ocular em $80 \%$ de pacientes com ceratoconjuntivites cicatriciais ${ }^{(9-10)}$. Em 1998, Tseng et al. estudaram o transplante de membrana amniótica em 31 olhos de 26

\begin{tabular}{|c|c|c|c|c|c|c|c|c|c|c|c|c|c|}
\hline Caso & Sexo & Idade & $\begin{array}{r}\text { Diagnóstico } \\
\text { pré } \\
\end{array}$ & $\begin{array}{l}\text { opessura } \\
\text { op corneal } \\
\text { escleral }\end{array}$ & $\begin{array}{l}\text { Tamanho do } \\
\text { afinamento } \\
\text { (milímetros) }\end{array}$ & $\frac{A V}{\text { pré-op p }}$ & $\frac{v}{\text { pós-op }}$ & Cirurgias & Resultado & $\begin{array}{l}\text { Espessura } \\
\text { pós-op } \\
\text { corneal } \\
\text { ou escleral }\end{array}$ & $\begin{array}{l}\text { Tempode } \\
\text { pós-op } \\
\text { (meses) }\end{array}$ & $\begin{array}{c}\text { Medicação } \\
\text { pós-op }\end{array}$ & $\begin{array}{l}\text { Epiteliza- } \\
\text { ção(dias) }\end{array}$ \\
\hline 1 & $M$ & 60 & $\begin{array}{l}\text { úlcera } \\
\text { neurotrófica } \\
\text { por HSV }\end{array}$ & $30 \%$ & $2 \times 2$ & MM & MM & $\begin{array}{l}\text { 1cirurgia } \\
\text { (2sp 1cp) }\end{array}$ & $S$ & $80 \%$ & 12 & $\begin{array}{l}\text { oflox } 4 x / d \\
\text { dexam } 4 x / d\end{array}$ & 20 \\
\hline 2 & $\mathrm{~F}$ & 53 & $\begin{array}{l}\text { úlcera neu- } \\
\text { rotrófica es- } \\
\text { tromal e epi- } \\
\text { telial pos HSV }\end{array}$ & $40 \%$ & $3 \times 1$ & CD3m & 0,15 & $\begin{array}{l}\text { 1cirurgia } \\
\text { (2sp 1cp) }\end{array}$ & $S$ & $90 \%$ & 12 & $\begin{array}{l}\text { oflox } 4 x / d \\
\text { dexam } 4 x / d\end{array}$ & 30 \\
\hline 3 & $\mathrm{~F}$ & 66 & $\begin{array}{l}\text { úlcera trófica } \\
\text { pós radioterapia } \\
\text { palpebral por } \\
\text { linfoma não } \\
\text { Hodgkin }\end{array}$ & $10 \%$ & $3 \times 3$ & MM & MM & $\begin{array}{l}3 \text { cirurgias: } \\
1^{a} \text { peritomia+ } \\
\text { membrana } \\
2^{a} \text { cirurgia } \\
\text { (2sp } 1 \mathrm{cp}) \\
3^{\text {a cirurgia }} \\
\text { (2sp } 1 \mathrm{cp})\end{array}$ & - & inalterada & $\begin{array}{l}15 \\
13 \\
12\end{array}$ & $\begin{array}{l}\text { tobra/dex } \\
4 / 4 \mathrm{~h} \\
\text { oflox3x/d } \\
+ \text { dexam } 2 x / d \\
\text { oflox3x/d } \\
\text { +dexam } 2 x / d\end{array}$ & $\begin{array}{l}\text { não epite- } \\
\text { lizou }\end{array}$ \\
\hline 4 & $\mathrm{~F}$ & 84 & $\begin{array}{l}\text { úlcera } \\
\text { trófica pós } \\
\text { transplante } \\
\text { de córnea }\end{array}$ & $30 \%$ & $3 \times 3$ & MM & MM & $\begin{array}{l}2 \text { cirurgias: } \\
\text { cola+LCT } \\
1 \text { cirurgia } \\
(2 \mathrm{sp} 1 \mathrm{cp})\end{array}$ & $S$ & $70 \%$ & 14 & $\begin{array}{l}\text { oflox } 3 x / d \\
\text { tobra/dex } \\
4 x / d\end{array}$ & 30 \\
\hline 5 & $\mathrm{~F}$ & 71 & $\begin{array}{l}\text { afinamento } \\
\text { escleral pós } \\
\text { betaterapia e } \\
\text { cirurgia de PT }\end{array}$ & $10 \%$ & $3 \times 3$ & 0,6 & 0,6 & $\begin{array}{l}\text { 1cirurgia } \\
\text { (2sp 1cp) }\end{array}$ & S & $70 \%$ & 14 & $\begin{array}{l}\text { tobra/dex } \\
4 / 4 \mathrm{~h} \\
\text { pilo } 5 x / d+ \\
\text { MT } 2 x / d\end{array}$ & 15 \\
\hline 6 & $\mathrm{~F}$ & 57 & $\begin{array}{l}\text { afinamento } \\
\text { escleral pós } \\
\text { betaterapia e } \\
\text { cirurgia de PT }\end{array}$ & $10 \%$ & $2 \times 2$ & 0,5 & 0,5 & $\begin{array}{l}\text { 1cirurgia } \\
\text { (2sp 1cp) }\end{array}$ & SP & $70 \%$ & 13 & $\begin{array}{l}\text { tobra/dex } \\
4 / 4 \mathrm{~h}\end{array}$ & 7 \\
\hline 7 & $\mathrm{~F}$ & 59 & $\begin{array}{l}\text { afinamento } \\
\text { escleral pós } \\
\text { cirurgia de PT }\end{array}$ & $10 \%$ & $4 \times 2,5$ & 0,6 & 0,6 & $\begin{array}{l}\text { 1cirurgia } \\
\text { (3sp 1cp) }\end{array}$ & $S$ & $90 \%$ & 11 & $\begin{array}{l}\text { tobra/dex } \\
4 / 4 \mathrm{~h}\end{array}$ & 10 \\
\hline \multicolumn{14}{|c|}{$\begin{array}{l}\text { M: masculino; F: feminino; Pré-op: pré-operatório; Pós-op: pós-operatório; AV: acuidade visual; MM: movimento de mão; CD: conta dedos; sp: sem pontos; cp: com } \\
\text { pontos; S: sucesso; SP: sucesso parcial; l: insucesso; oflox: ofloxacina 0,3\%; dexam: dexametasona 0,005\%; Tobra/dex: Tobramicina 0,3 mg/ml+dexametasona1mg/ } \\
\text { ml; Pilo: pilocarpina1\%; MT: maleato de Timolol; m: metros; LCT: lente de contato terapêutica; Cola: adesivo tecidual de cianoacrilato; HSV: herpes simples virus; } \\
\text { PT: pterígio; h: horas; x/d: vezes ao dia }\end{array}$} \\
\hline
\end{tabular}


pacientes com deficiência limbar leve, moderada e severa ${ }^{(7)}$. A epitelização completa ocorreu em 93,5\% dos pacientes (2 a 4 semanas), sendo que $83 \%$ dos pacientes apresentaram melhora da acuidade visual ${ }^{(7)}$.

Defeitos epiteliais crônicos constituem complicações de difícil resolução nas afecções severas da superfície ocular, que podem evoluir para inflamação estromal e infecção. A fisiopatologia associa aspectos não-inflamatórios, como olho seco, exposição, triquíase e condições corneais neurotróficas, a aspectos inflamatórios, com a presença de leucócitos polimorfonucleares ou seus produtos inibindo o processo de cicatrização epitelial ${ }^{(11,18)}$. Diversos tratamentos são relacionados na literatura, como o uso de medicação tópica (lubrificantes sem preservativos, esteróides tópicos, antibióticos), lente de contato terapêutica, tarsorrafia e recobrimento conjuntival ${ }^{(17)}$. Lee e Tseng relataram bons resultados com transplante de membrana amniótica em 10 de 11 casos de defeito epitelial crônico de diferentes etiologias ${ }^{(11)}$. Em 4 olhos houve dissolução da brana amniótica, o que não impediu que ocorresse cicatrização epitelial da mesma maneira do que nos casos em que a membrana amniótica foi incorporada no estroma ${ }^{(11)}$. Esse achado sugere que a membrana possui diferentes mecanismos de ação que estimulam a epitelização(11). Em 1999, Kruse et al. propuseram a utilização de várias camadas de membrana amniótica para a reconstrução de úlceras estéreis de córnea ${ }^{(19)}$. Os autores observaram cicatrização epitelial em todos os casos e estabilidade da reconstrução em 9 dos 11 casos. Após o procedimento, os autores notaram que as membranas gradualmente se dissolveram num período de 12 meses de seguimento.

Afinamento escleral constitui fator predisponente para infecção, perfuração, ectasia e exposição escleral e severa reação de câmara anterior. Lin et al. utilizaram esclera preservada com adesivo tecidual em pacientes que com afinamento escleral, referindo ótimos resultados no restabelecimento da espessura do local ${ }^{(20)}$.

No presente trabalho, realizamos transplante de membrana amniótica com a função de promover a epitelização e restabelecer a espessura de afinamentos córneo-esclerais. Nesses casos, a membrana deve idealmente ser colocada com a face epitélio/membrana basal para cima, pois se objetiva seu recobrimento pelas células epiteliais corneais ou conjuntivais adjacentes. Como a membrana amniótica é formada basicamente por colágeno, seu uso para o preenchimento de áreas afinadas permite que ocorra aumento da espessura corneal ou escleral quando incorporada. Nos casos de afinamento corneal, obtivemos bons resultados em 3 pacientes (75\%). O caso que não foi bem sucedido referia-se a uma paciente com úlcera neurotrófica após irradiação palpebral. Apesar da paciente ter evoluído com defeito epitelial persistente, a mesma relatava melhora significativa nos sintomas de dor e fotofobia intensas. Lee e Tseng também obtiveram o mesmo resultado em caso semelhante ${ }^{(11)}$. Aparentemente, o efeito da membrana amniótica nesses casos foi, além da restauração da estrutura da córnea, o de diminuir o processo inflamatório e estimular o epitélio por suas propriedades bioquímicas já descritas anteriormente. Nos afinamentos esclerais (3 casos), observamos bons resultados, com restabelecimento da sua espessura e bom aspecto estético. Na literatura compilada, só há 1 relato de uso de membrana amniótica para afinamento escleral, porém acompanhada de utilização de esclera preservada como manchão( ${ }^{(21)}$.

\section{CONCLUSÕES}

Todos esses argumentos indicam que a membrana amniótica constitui uma opção alternativa de grande utilidade na reconstrução da superfície ocular e restauração da espessura nos casos de afinamento corneais e esclerais. No entanto, a associação com outras modalidades terapêuticas parece ser necessária nos casos severos de úlceras neurotróficas de córnea, especialmente após radiação. Estudos com maior casuística, prospectivos e randomizados serão necessários para melhor avaliar esse procedimento.

\section{AGRADECIMENTOS}

Ophthalmos, São Paulo/SP; Hospital Maternidade São Luiz, São Paulo/SP.

\section{ABSTRACT}

Purpose: To evaluate the use of amniotic membrane (AM) as an adjunct to the treatment of corneal and scleral thinnings. Methods: Amniotic membrane was obtained at the time of cesarean section and was preserved at $-80^{\circ} \mathrm{C}$ in glycerol and cornea culture media at a ratio of 1:1. Seven eyes from 7 patients were divided into Group 1: neurotrophic ulcers secondary to herpes simplex virus keratitis ( 2 cases), after corneal transplantation (1 case), and post palpebral radiation ( 1 case); and Group 2: scleral thinning after pterygium surgery with (2 cases) or without (1 case) beta-therapy. All cases underwent amniotic membrane transplantation to restore thickness and promote epithelialization. Results: Mean follow-up time was 12 months (range, 11-15 months). One case of neurotrophic ulcer secondary to radiotherapy was unsuccessful. A successful outcome was observed in the other 6 cases. Regarding visual acuity, 1 case improved and the other 6 cases remained unchanged. The mean epithelialization time was $26.6 \pm 5.8$ days for group 1 and $10.6 \pm 4.0$ days for group 2. Conclusions: The use of amniotic membrane represents a useful alternative to ocular surface reconstruction in corneal and scleral thinning. More cases and a longer follow up are necessary to better evaluate this procedure.

Keywords: Amnion/transplantation; Fetal tissue transplantation; Tissue preservation; Homologous transplantation; Cornea/surgery; Cornea/pathology; Conjunctiva/transplantation; Sclera/surgery; Corneal epithelium; Follow-up 


\section{REFERÊNCIAS}

1. Trelford JD, Trelford-Sauder M. The amnion in surgery, past and present. Am J Obstet Gynecol 1979;134:833-45.

2. Colocho G, Graham WP, Greene AE, Matheson DW, Lynch D. Human amniotic membrane as a physiologic wound dressing. Arch Surg 1974;109:370-3.

3. Kim JC, Tseng SC. Transplantation of preserved human amniotic membrane for surface reconstruction in severely damaged rabbit corneas. Cornea 1995; 14:473-84.

4. Tsubota K, Satake Y, Ohyama M, Toda I, Takano Y, Ono M, et al. Surgical reconstruction of the ocular surface in advanced ocular cicatricial pemphigoid and Stevens-Johnson syndrome [commented on Am J Ophthalmol 1996; 122:914-5]. Am J Ophthalmol 1996;122:38-52.

5. Talmi YP, Sigler L, Inge E, Finkelstein Y, Zohar Y. Antibacterial properties of human amniotic membranes. Placenta 1991;12:285-6.

6. Adinolfi M, Akle CA, McColl I, Fenson AH, Tansley L, Connolly P, et al. Expression of HLA antigens, $b_{2}$-microglobulin and enzymes by human amniotic epithelial cells. Nature 1982;295:325-7.

7. Tseng SC, Prabhasawat P, Barton K, Gray T, Meller D. Amniotic membrane transplantation with or without limbal allografts for corneal surface reconstruction in patients with limbal stem cell deficiency. Arch Ophthalmol 1998;116:431-41.

8. Shimazaki J, Yang HY, Tsubota K. Amniotic membrane transplantation for ocular surface reconstruction in patients with chemical and thermal burns [commented on Ophthalmology 2000;107:411-2]. Ophthalmology 1997; 104:2068-76, 1997.

9. Gomes JAP, Komagome CM, Santos N, Cunha MC, Freitas D. Membrana amniótica nas cirurgias reconstrutivas da superfície ocular nas ceratoconjuntivites cicatriciais. Arq Bras Oftalmol 1999;62:562-76.

10. Gomes JAP. Atualização no tratamento das ceratoconjuntivites cicatriciais. Arq Bras Oftalmol 2000;63:91-6.
11. Lee S, Tseng SC. Amniotic membrane transplantation for persistent epithelial defects with ulceration. Am J Ophthalmol 1997;123:303-12.

12. Prabhasawat P, Barton K, Burkett G, Tseng SC. Comparison of conjunctival autografts, amniotic membrane grafts and primary closure for pterygium excision. Ophthalmology 1997;104:974-85.

13. Tseng SC, Prabhasawat P, Lee SL. Amniotic membrane transplantation for conjunctival surface reconstruction. Am J Ophthalmol 1997;124:765-74.

14. Barton K, Budenz DL, Khaw PT, Tseng SCG. Amniotic membrane transplantation in glaucoma surgery [abstract]. Invest Ophthalmol Vis Sci 1997;38 (4 Pt 2):S473.

15. Choi YS, Kim JY, Wee WR, Lee JH. Effect of the application of human amniotic membrane on rabbit corneal wound healing after excimer laser photorefractive keratectomy. Cornea 1998;17:389-95.

16. Tseng SC, Tsubota K. Important concepts for treating ocular surface and tear disorders. Am J Ophthalmol 1997;124:825-35.

17. Wagoner MD, Kenyon KR, Gypson IK, Hanninen LA, Seng WL. Polymophonuclear neutrophilis delay corneal epitelial wound healing in vitro. Invest Ophthalmol Vis Sci 1984;25:1217-20.

18. Prabhasawat P, Tseng SC. Impression cytology study of epitelial phenotype of ocular surface reconstructed by preserved human amniotic membrane. Arch Ophthalmol 1997;115:1360-7.

19. Kruse FE, Rohrschneider K, Volcker HE. Multilayer amniotic membrane transplantation for reconstruction of deep corneal ulcers. Ophthalmology 1999; 106:1504-11; discussion 1511.

20. Lin CP, Tsai MC, Wu YH, Shih MH. Repair of a giant scleral ulcer with preserved sclera and tissue adhesive. Ophthalmic Surg Lasers 1996;27:995-9.

21. Rodrigues -Ares MT, Touriño R, Capeans C, Sanches-Salorio M. Repair of scleral perforation with preserved scleral and amniotic membrana in Marfan's syndrome. Ophthalmic Surg Lasers 1999;30:485-7. 